\title{
Similarities between insect swarms and isothermal globular clusters
}

\author{
Dan Gorbonos, ${ }^{1, *}$ Kasper van der Vaart, ${ }^{2, \dagger}$ Michael Sinhuber, ${ }^{2}$ James G. Puckett, ${ }^{3}$ Andrew M. Reynolds, ${ }^{4}$ \\ Nicholas T. Ouellette, ${ }^{2}$ and Nir S. Gov $\mathbb{1}^{1, 末}$ \\ ${ }^{1}$ Department of Chemical and Biological Physics, The Weizmann Institute of Science, P.O. Box 26, Rehovot 76100, Israel \\ ${ }^{2}$ Department of Civil and Environmental Engineering, Stanford University, Stanford, California 94305, USA \\ ${ }^{3}$ Department of Physics, Gettysburg College, Gettsyburg, Pennsylvania 17325, USA \\ ${ }^{4}$ Rothamsted Research, Harpenden AL5 2JQ, United Kingdom
}

(Received 10 November 2019; accepted 6 February 2020; published 6 March 2020)

\begin{abstract}
Previous work has suggested that disordered swarms of flying insects can be well modeled as self-gravitating systems, as long as the "gravitational" interaction is adaptive. Motivated by this work, we compare the predictions of the classic, mean-field King model for isothermal globular clusters to observations of insect swarms. Detailed numerical simulations of regular and adaptive gravity allow us to expose the features of the swarms' density and velocity profiles that are due to long-range interactions and are captured by the King model phenomenology, and those that are due to adaptivity and short-range repulsion. Our results provide further support for adaptive gravity as a model for swarms.
\end{abstract}

DOI: 10.1103/PhysRevResearch.2.013271

\section{INTRODUCTION}

Insect swarms are a canonical example of collective animal behavior [1,2], displaying group-level cohesion and stability even in the presence of environmental noise [3-8]. But while in many other forms of collective animal motion, such as flocking, schooling, and herding [2], the movement of individuals is coordinated, swarms are distinguished by their lack of globally aligned motion and the swarm state is not described by any order parameter.

It is thought that many swarming insect species such as Chironomus riparius, the midge species we consider here, interact predominantly via long-range acoustic sensing [9]. Indeed, a theoretical model that assumes that midges accelerate toward the sounds produced by other individuals has produced nontrivial results that are in good agreement with empirical observations $[10,11]$. The acoustic field produced by flying insects has a monopole component whose intensity falls off according to an inverse-square law [12], a scaling that is similar to the way the gravitational pull between objects falls off with distance. Dipole and other higher-order multipole components decay more rapidly, and are thus weaker than the monopole component. It is therefore tempting to speculate, as Okubo [3] and then Gorbonos et al. [10] did, that midge swarms are analogous to $N$-body self-gravitating systems,

\footnotetext{
*Present address: Max Planck Institute of Animal Behavior, Dept. of Collective Behavior, Universitätsstraße 10, 78464 Konstanz, Germany.

†resent address: Max Planck Institute for Dynamics and SelfOrganization, 37077 Göttingen, Germany.

ॠnir.gov@weizmann.ac.il

Published by the American Physical Society under the terms of the Creative Commons Attribution 4.0 International license. Further distribution of this work must maintain attribution to the author(s) and the published article's title, journal citation, and DOI.
}

where cohesion originates from the gravitational pull between the bodies compromising the system. A key contribution from Ref. [10] was to incorporate the adaptive gain of typical biological sensors, which leads to results that are different from Newtonian gravitation but are in good agreement with empirical measurements of swarms.

This adaptive-gravity model was shown in Ref. [10] to capture many global features of midge swarms. For example, it correctly reproduces the linear radial dependence of the mean acceleration of midges toward the swarm center, the scaling of this acceleration with the swarm size, and the viriallike relation between the mean kinetic and potential energies of the whole swarm.

The analogy between swarms and self-gravitating systems is further appealing because it is well known that gravity can produce complex dynamical behavior from simple interactions-just as is thought to be the case for collective animal behavior [13]. Thus, by making a link between these two distinct systems, we can draw on the intuition built up from studying gravity to gain insight into collective animal behavior.

In this paper, we examine this analogy more closely by studying the spatial variation of the number density and the velocity of individuals. We compare to the classic King model for the mass distribution in isothermal globular clusters [14] (see Appendix A). Although we find some similarities with the observed swarms, there are also significant differences. These discrepancies reveal the limitations of a pure gravitational model for swarms. Furthermore, we aim to explore which of the observed global features of the midge swarm arise from the long-range nature of the interactions and which depend also on the property of adaptivity. Long-range interactions are of course a feature of regular gravity, and therefore also appear in globular clusters, which are well described by the King model. We would then expect that features that are crucially dependent on adaptivity, which is not present in regular gravity, will be missing from a 
King-model description of the swarm. We find that the addition of adaptivity, which we can introduce only using numerical simulations, together with short-range repulsion, produces mass distributions and dynamics that capture all the main features of the midge swarm data. Thus, our results provide significant further support for adaptive gravity as a model for the swarm behavior.

\section{DENSITY PROFILES}

\section{A. Density profiles: King's model and midge swarms}

We begin by considering the mass distributions predicted for regular gravity (such as in globular clusters) by the original King model. This model has been well studied previously. Chavanis et al. [15], for example, described the solution space for globular clusters by fixing the cluster mass and varying the other system parameters, such as temperature, cluster radius, energy, and so forth. To adapt this model for swarms, we use a fixed inverse temperature $\beta$, which describes isothermal globular clusters. In addition to being simple to implement (see Appendix A), this choice is motivated by the observation that swarms of different sizes empirically display roughly the same amount of kinetic energy per midge [16,17]. For large globular clusters, a King model with fixed temperature gives a roughly constant kinetic energy per particle, independent of system size (Fig. 4). With this assumption, we compute the density profiles predicted by the King model [Figs. 1(a) and 1(b)], using Eqs. (A15) and (A16). Note that for easier comparison between the different models, we plot the density profiles for all the models in Fig. 1, and the corresponding quantitative measures in Fig. 2.

The King model predicts two distinct branches of solutions, which are clearly observed when we plot quantitative measures of the distributions such as the total mass $M$ [Fig. 2(a), where we assume that all midges have the same mass], the density at the center $\rho_{0}$ [Fig. 2(b)], and the kurtosis [Fig. 2(c)] as functions of the overall swarm size $R_{s}$. Here, $R_{s}$ is defined as the mean distance of a midge from the center of mass of the swarm: $R_{s} \equiv \int_{0}^{\infty} r^{3} \rho(r) d r / \int_{0}^{\infty} r^{2} \rho(r) d r$, where $\rho(r)$ is the density profile. The two branches are termed stable and unstable [shown in blue and red, respectively, in Figs. 2(a)-2(c)], and are distinguished by the sign of the heat capacity (positive or negative, respectively) of the cluster in the canonical ensemble [15]. It is found empirically that globular clusters reside on the unstable branch [15], presumably due to the strong destabilizing effects of "slingshots"- that is, anomalously high acceleration events that arise due to close encounters.

In Figs. 1(c) and 1(d), we plot the empirical density profiles measured for laboratory midge swarms. Details of the laboratory setup and measurement protocols are given in Refs. $[4,18]$. The primary difference between the data in Figs. 1(c) and 1(d) is that the swarms in Fig. 1(c) were observed in a cubical laboratory enclosure measuring $91 \mathrm{~cm}$ on a side, while the enclosure for the swarms in Fig. 1(d) measured $122 \mathrm{~cm}$ on a side.

Qualitatively, the density profiles from the swarms are similar in many aspects to those computed from the King model, though the agreement is not exact. To illuminate these similarities and differences further, we computed the same

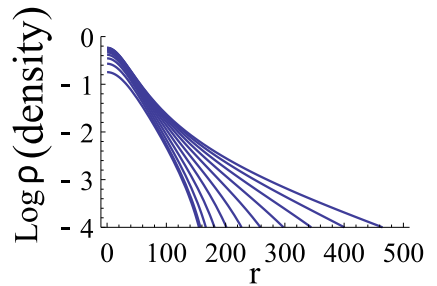

(a)

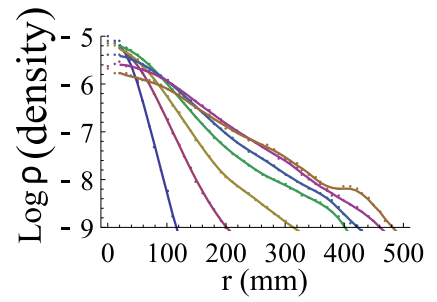

(c)

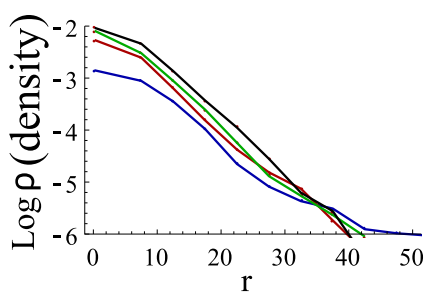

(e)

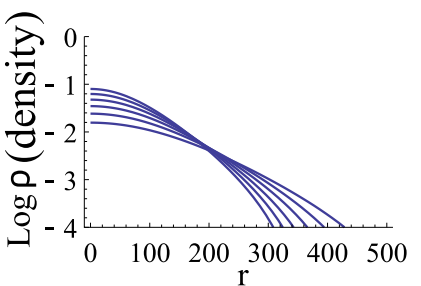

(b)

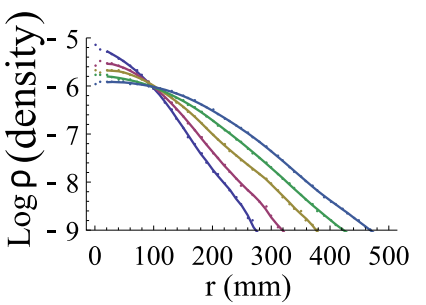

(d)

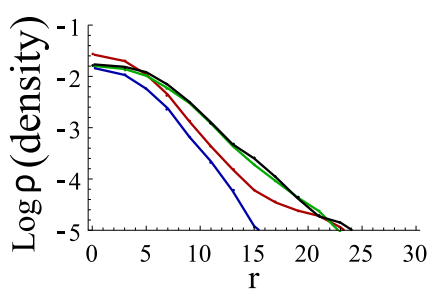

(f)
FIG. 1. (a), (b) Two families of density profiles computed from the King model [Eqs. (A15) and (A16)] for different initial conditions, with shapes that are qualitatively similar to results from real swarms (c), (d). (a) corresponds to the unstable branch and (b) to the stable branch (see the discussion in the text). (c), (d) Two families of density profiles measured in real swarms in two different laboratory setups (see text). (e) Density profiles of simulated swarms using the adaptive gravity model. Simulations were run using $N=12$ and $R_{s}=16.2$ (blue), $N=24$ and $R_{s}=11.8$ (red), $N=32$ and $R_{s}=$ 11.5 (green), and $N=48$ and $R_{s}=12$ (black). (f) Density profiles of simulated swarms including adaptivity and short-range repulsion. Simulations were run using $N=12$ and $R_{s}=6$ (blue), $N=24$ and $R_{s}=6.7$ (red), $N=32$ and $R_{s}=8.2$ (green), and $N=48$ and $R_{s}=8.1$ (black).

distribution measures for the swarms as for the King model, shown in Figs. 2(d)-2(f). The density at the center of the midge swarm $\left(\rho_{0}\right)$ was obtained in Figs. 2(e), 2(h), and 2(k) by fitting a Gaussian to the density profile near the center of mass. We see in Fig. 2(e) that $\rho_{0}$ slightly decreases with increasing swarm size, which qualitatively fits the stable branch of the King model (where this decrease is much steeper). The total number of midges (as a proxy for the total mass) increases slowly with the swarm radius, a feature exhibited by the unstable branch of the King model (the stable branch has the opposite relation).

The kurtosis of the midge swarms falls in a range of values between the King model branches and is significantly larger (i.e., the swarms have heavier tails than they would if they were Gaussian) than the density profiles along the King model stable branch (which are very close to Gaussian). Note that the kurtosis is a standard measure to quantify simple but non-Gaussian distribution shapes (as long as they are 


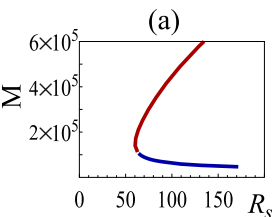

(d)

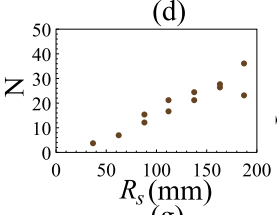

(g)

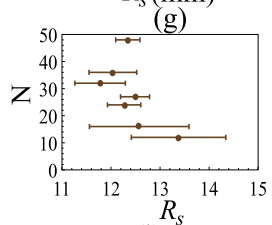

(j)
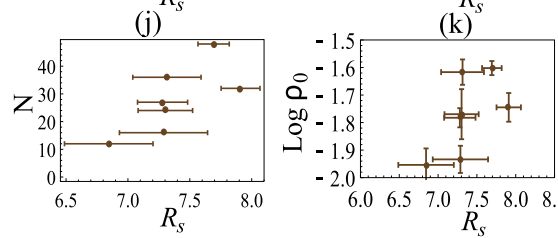

(b)

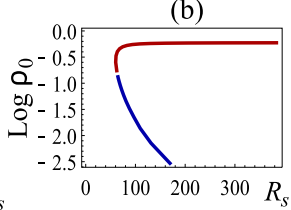

(e)

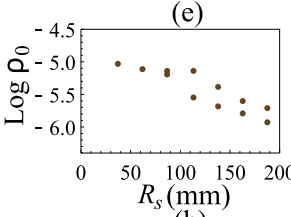

(h)

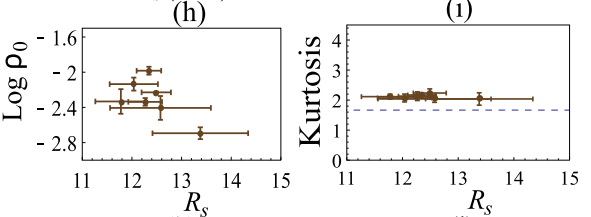

(1)

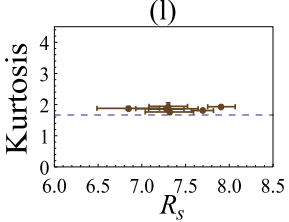

FIG. 2. (a)-(c) Total mass $M$ (a), density at the center $\rho_{0}$ (b), and kurtosis (c) as a function of the swarm size $R_{s}$ for a series of King model solutions. The blue line corresponds to the stable branch in the canonical ensemble and the red one to the unstable one. The kurtosis of a Gaussian distribution in three dimensions, $5 / 3$, is denoted by the horizontal dashed line. (d)-(f) The same quantities computed from the measured data for real swarms. (g)-(i) The same quantities computed for simulated swarms with adaptivity. (j)-(l) The same quantities computed for simulated swarms with adaptivity and short-range repulsion.

monotonically decreasing functions, as shown in Fig. 1). Thus, it provides an interpretable way of comparing the different models, and highlights discrepancies between the midge swarms and the King model behavior. Furthermore, the density profiles for standard gravity (King's model) are non-Gaussian.

\section{B. Density profiles: Modified gravity models}

To attempt to reconcile the model predictions with the empirical results for real swarms, we consider two modifications to normal Newtonian gravity. First, since particles in the simulations have a strong tendency to develop anomalously high accelerations due to slingshots and thus to evaporate from the cluster, it is common to include a softening parameter $\epsilon$ (with units of length) to the gravitational force [19], so

$$
\vec{F}_{\epsilon, g}^{i}=C \sum_{j} \hat{r}_{i j} \frac{1}{\left|\vec{r}_{i}-\vec{r}_{j}\right|^{2}+\epsilon^{2}},
$$

where $C$ is a constant with units of (force $\times$ length $^{2}$ ), $\vec{r}_{i}$ is the position vector for midge $i$, and $\hat{r}_{i j}$ is the unit vector pointing from midge $i$ to midge $j$. This "epsilon" gravity modifies the force at short distances, up to $\left|\vec{r}_{i}-\vec{r}_{j}\right|=O(\epsilon)$. We chose a value of $\epsilon=\sqrt{15} \simeq 3.87$, so it is smaller than the mean swarm size $R_{s}$ (Figs. 1 and 15) but is still effective in preventing slingshots and maintaining a stable swarm.
In addition, we simulated our previously introduced adaptive-gravity model [10]. In this case, the effective force felt by midge $i$ due to midge $j$ is given by

$$
\vec{F}_{\text {eff }}^{i}=C \sum_{j} \frac{\hat{r}_{i j}}{\left|\vec{r}_{i}-\vec{r}_{j}\right|^{2}+\epsilon^{2}} \frac{R_{\mathrm{ad}}^{-2}}{R_{\mathrm{ad}}^{-2}+\sum_{k}\left(\left|\vec{r}_{i}-\vec{r}_{k}\right|^{2}+\epsilon^{2}\right)^{-1}} .
$$

The logic underlying this model is that the strength of the signal received by each midge should be renormalized by the total buzzing noise due to all the other midges, to mimic the typical adaptive gain of biosensors [20]. We define a length scale, $R_{\text {ad }}$, over which this adaptivity occurs, and when $r_{i j} \gg \sqrt{N} R_{\mathrm{ad}}$, such that the distance between a pair of midges is much larger than the adaptive range, the model reduces to epsilon gravity (which itself reduces to Newtonian gravity when $\epsilon=0$ ). Within the range of adaptivity, where the midges are close to each other compared to $R_{\mathrm{ad}}$, the term $R_{\mathrm{ad}}^{-2}$ is negligible in the denominator of the second factor in Eq. (2), and we see that the vectorial sum of the first factor is simply divided by the scalar sum in the second factor. This gives rise to what we term perfect adaptivity, where the response does not depend on the absolute intensity of the background signal.

Finally, we also simulated the adaptive-gravity model with an additional short-range repulsion between the midges. This effect was empirically observed and measured in real swarms [21], and was implemented in an earlier simulation scheme [10]. This type of effective interaction arises due to midges avoiding collisions while flying. Here we use a repulsive force with a cutoff to capture this effect, given by

$$
\vec{F}_{\text {rep }}^{i}=\left\{\begin{array}{cc}
C \sum_{j}\left(\frac{1}{L_{c}^{2}}-\frac{1}{\left|\vec{r}_{i}-\vec{r}_{j}\right|^{2}}\right) \hat{r}_{i j} & \left|\vec{r}_{i}-\vec{r}_{j}\right|<L_{c} \\
0 & \left|\vec{r}_{i}-\vec{r}_{j}\right| \geqslant L_{c},
\end{array}\right.
$$

where we take $L_{c} \approx 0.2 R_{\text {ad }}$. We chose this value as it was the minimal value for $L_{c}$ that produced a noticeable effect on the qualitative features of the swarms, as compared with those without repulsion (see below). Larger values of $L_{c}$ would make the repulsion force dominant in small, finite-size swarms, and we wanted to stay in the regime where the long-range gravitylike interactions play the dominant role for the large-scale organization of the swarm. In the simulations, we worked in the regime where $R_{s} / L_{c} \sim 4$, which is within the range observed in the experiments $R_{s} / L_{c} \sim 4-16$ [21].

With these interaction laws, we considered several different swarm sizes $R_{s}$, as defined above. We used $R_{\text {ad }}=10 \approx R_{S}$, placing us in the regime where adaptivity affects the vast majority of the midges in the swarm. Unlike for regular gravity, where we can use the coarse-grained King model, for adaptive-gravity we do not have at present an equivalent coarse-grained description. Therefore, to explore the spatial distribution of mass in the adaptive gravity model, we turned to numerical simulations. We used a scheme for performing $N$-body dynamics originally developed by Aarseth (see Appendix 4.B of Ref. [22]), which allows for accurate numerical integration using fourth-order equations of motion. A complete description of the numerical method is given in Ref. [19]. The acceleration of each mass in the simulation is computed by the direct summation of the forces due to the other $N-1$ bodies following the form of the force law. The 


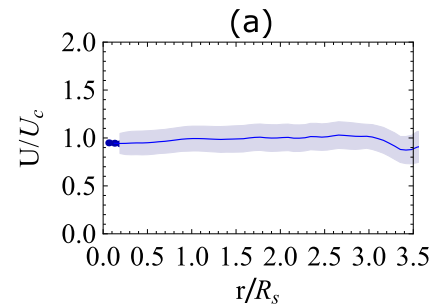

(c)

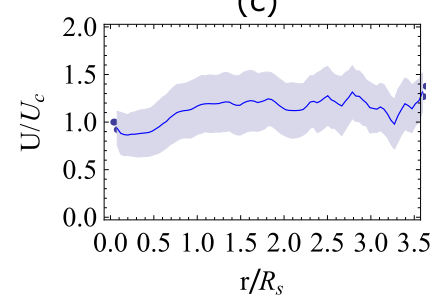

FIG. 3. Mean speed as a function distance from the center of the swarm, normalized by the speed at the center. Data are shown for (a) real laboratory swarms, (b) epsilon-gravity, (c) adaptive gravity, and (d) adaptive gravity with short-range repulsion. The brown curves in (b) correspond to a family of King model solutions [Figs. 1(a) and 1(b), computed numerically according to Eq. (A23)]. In (d), we truncated the plot at $r \sim 2 R_{s}$ since outside this radius the particles are not part of the cohesive region (i.e., the swarm) (see Fig. 12).

scheme was designed to work efficiently for up to $N=50$, well within the range of typical midge swarms [4,18]. For further details about the simulation see Appendices B, F, and G. Initially, particles were placed randomly in a simulation box of varying side lengths to control the kinetic energy of the system. This was achieved (except for the epsilongravity system; see below) by varying the initial conditions of the simulations until the kinetic energy per particle was within $10 \%$ of the desired value in the long-time limit (Fig. 13). The initial velocities were zero, but under the influence of the forces the particles accelerated and eventually reached a quasistationary state (Figs. 5-7) that exhibits the distinct non-Gaussian velocity statistics (Fig. 8) observed in real swarms [4]. Note that we do not include explicit noise in the simulations, and the trajectories become ergodic due to the natural tendency of $N$-body gravitating systems to be chaotic.

We now compare the same quantitative features of the density profiles computed from the numerical simulations to those from the King model and the empirical swarm measurements. Note that the theoretical calculations of the King model and the simulations are done in arbitrary units of length, while the experimental measurements have an inherent length scale (Figs. 1 and 2). Wherever possible we normalized the axes to become dimensionless (such as in Figs. 3, 10, and 11), but it is not always obvious how to do so.

We start with the epsilon-gravity interaction [Eq. (1), using $\epsilon=3.8$, which was much smaller than the swarm size $R_{s}$; Fig. 15]. Not surprisingly, some measures [Figs. 15(b) and 15 (c)] seem to agree well with the stable branch of the King model [Figs. 2(a)-2(c)], since close-encounter slingshots are greatly suppressed by the softened gravity. Other similarities include a decrease in the size $R_{s}$ and sharp decrease in $\rho_{0}$ for larger swarms.
Comparing to real midge swarms, we find that the decrease in $\rho_{0}$ for larger swarms in epsilon-gravity [Fig. 15(c)] is qualitatively similar, though somewhat stronger [Fig. 2(e)]. This similarity suggests that this feature arises from the longrange character of the interactions between the midges and that adaptivity only acts to weaken it (see below). The epsilongravity-force law does not agree very well with another observed feature of the midge swarms [Fig. 2(d)], where the size increases with the number of particles. Note that for epsilon gravity, we were not able to keep the kinetic energy per particle constant when changing the overall number of particles in the swarm (Fig. 14).

Figures 1(e) and 2(g)-2(i) show the results from adaptivegravity simulations [Eq. (2), using $R_{\mathrm{ad}}=10$ ]. Note that the swarms cover a much smaller range of sizes $\left(R_{S}\right)$, compared to the real [Figs. 1(c), 1(d), and 2(d)-2(f)] or epsilon-gravity swarms (Fig. 15). This is due to the sharp increase in the density at the center when the number of particles increases combined with the fixed kinetic energy per particle, thereby maintaining a roughly constant $R_{s}$. We find that $\rho_{0}$ decreases with increasing $R_{s}$, similar to the observations in real swarms (as well as epsilon-gravity and the stable branch of the King model). The kurtosis is similar to the values seen in the real swarms, and in standard gravity [King's model, Fig. 2(c)]. We therefore conclude that the long non-Gaussian tails in the density profiles arise from the long-range interactions and do not require the adaptivity property.

However, just as for epsilon gravity and the stable branch of the King model, the biggest discrepancy with the real swarms is the relation between the number of particles (midges) and $R_{s}$, which we find in the simulations to be a decreasing function [Fig. $2(\mathrm{~g})$ ] even though it is increasing in real swarms [Fig. 2(d)].

We therefore also tested the addition of a short-range repulsion [Eq. (3)] to the adaptive-gravity simulation [Figs. 1(f) and 2(j)-2(l)]. This additional ingredient makes $\rho_{0}$ roughly independent of $R_{s}$ while not changing the kurtosis significantly. Both measures are in reasonable agreement with the real swarm data, considering the small range of swarm sizes in the simulations. The primary change as a result of the shortrange repulsion is the appearance of an overall increasing relation between the number of particles and swarm size [Fig. 2(j)]. This feature, which is observed in the real swarms [Fig. 2(d)], is absent from all the simulations that contain only long-range attractive interactions. This model therefore captures qualitatively all the main features of the density profiles of the midge swarms.

These results allow us to clearly delineate the properties of the midge swarm that are due to the long-range (adaptive) gravity interactions, namely, the large kurtosis (heavy tails) and a slow decrease of the density at the swarm center for increasing swarm sizes. The short-range repulsion is responsible for inflating the size $\left(R_{S}\right)$ of swarms with increasing number of midges, which is counter to the behavior of purely long-range interactions (both epsilon and adaptive gravity).

\section{VELOCITY PROFILES}

So far, we have considered the spatial density distribution of the swarm. We now turn to the spatial distribution of the 
velocities of the particles in the swarm, as another way to distinguish between the different models and compare to the observations from real swarms. We therefore computed the average speed of midges as a function of the distance $r$ away from the center of mass of the swarm using the dataset from the larger midge enclosure [18]. As shown in Fig. 3(a), these speeds are essentially independent of position (as also noted in Ref. [17]). Thus, the mean kinetic energy of a midge is also statistically uniform in space [Fig. 11(a)].

The simulated speed profile for epsilon-gravity is shown in Fig. 3(b). We see that the velocity decreases rapidly with increasing radius from the swarm center, in good agreement with the profiles calculated from the King model but contrary to the data for real swarms. By contrast, the velocity profile computed using adaptive gravity [Fig. 3(c)] is rather flat, with a small increase of speed for small $r$, presumably because the potential is too strongly softened in the swarm center. The velocity profile becomes even smoother when short-range repulsion is included [Fig. 3(d)], and is similar to the empirical results for real swarms. We find similar results for the standard deviation of the speed (Fig. 11).

The uniformity of the velocity profile across the swarm (Figs. 3 and 11) serves as strong support for the adaptivegravity model. Note that the midges do not move at constant instantaneous speed when flying through the swarm, and that the global velocity distribution has long (nearly exponential) tails (see Ref. [4] and Fig. 8), so it is not obvious a priori that there would not be some radial dependence in the local velocity distribution. Therefore, the novel observation of a flat velocity distribution [shown in Figs. 3(a) and 11(a)] in midge swarms is a challenge that must be met by any theoretical model. We demonstrate that this property does not arise in the simplest gravitational model (King model) and so it is not a consequence of long-range interactions alone. The additional property of adaptivity, however, recovers this characteristic, and we therefore view this as strong support for this model.

Finally, we considered the relation between mean acceleration and speed for swarms of different sizes (Fig. 10) [17]. We find that epsilon-gravity fails to reproduce the observed collapse of the curves for all swarm sizes, while the adaptive gravity captures this relation extremely well. This agreement gives added support to the adaptive-gravity model. It demonstrates that this model accounts for this observation, and there is no need for additional (velocity-dependent) interactions [17].

\section{CONCLUSION}

Taken together, our results provide further evidence that the interactions in insect swarms are well described by the adaptive-gravity framework, together with a short-range repulsion. This highlights that two key ingredients-long-range interactions and adaptivity - are essential. This model naturally captures many of the unusual properties of swarms, some of which have surprising similarities to globular clusters. Adaptivity, however, also induces additional effects. When the binding to the swarm is adaptive, the forces on one midge due to the other midges are not additive, and as a consequence the sum of the forces felt by all of the midge need not vanish, as it must for Newtonian gravity [10]. The center of mass of the swarm can therefore experience accelerations [23]. Such fluctuations have the potential to change fundamentally the characteristics of individual flight patterns; for example, Reynolds and Ouellette [23] showed that center of mass fluctuations allow for the emergence of Lévy flight patterns. Center-of-mass movements may also help to stabilize insect swarms against environmental perturbations [24].

These results suggest that it may be fruitful in the future to consider modifications of the interactions within the King model as a general framework for describing the behavior of active systems with long-range interactions, whatever their form. The proper analytical treatment of a King model augmented with an adaptive-gravity interaction law remains a challenge for future studies. Finally, future work on swarm modeling in particular should explore the effects of adding self-propulsion and stochasticity (noise), both of which are absent from the current models but certainly present for real insects. This kind of more realistic description of the motion of individual midges may also be required for the theory to describe the response of the midge swarm to external perturbations $[7,8]$, and in particular the dynamics of this response.

\section{ACKNOWLEDGMENTS}

We thank Tsvi Piran, Sverre Aarseth, and Tal Alexander for useful discussions. The research at Stanford was sponsored by the Army Research Laboratory and accomplished under Grant No. W911NF-16-1-0185. The views and conclusions in this document are those of the authors and should not be interpreted as representing the official policies, either expressed or implied, of the Army Research Laboratory or the U.S. Government. K.v.V. acknowledges support from an Early Postdoc Mobility fellowship from the Swiss National Science Foundation, and M.S. acknowledges support from the Deutsche Forschungsgemeinschaft under Grant No. 396632606. The work at Rothamsted forms part of the Smart Crop Protection (SCP) strategic program (No. BBS/OS/CP/000001) funded through the Biotechnology and Biological Sciences Research Council's Industrial Strategy Challenge Fund.

\section{APPENDIX A: THE KING MODEL}

Most globular clusters are well fit by the King model [14], as it captures their basic structure and main observed features [15]. This model is one of a series of models that assume a Maxwell-Boltzmann distribution of the velocities, although it is well-known that a self-gravitating system cannot actually be in thermodynamic equilibrium. This fact is a result of the long-range nature of the interactions and the Boltzmann entropy, which has no maximal value in an unbounded domain. Therefore, self-gravitating systems, as part of their out-of-equilibrium nature, have a strong tendency to evaporate over time. Since evaporation is a slow process, the system can be considered to be in a quasistationary state for times that are much shorter than the evaporation time, and the Maxwell-Boltzmann distribution can therefore serve as a good approximation.

To write a quasistationary expression for the distribution $f(\vec{r}, \vec{v})$ (that is, as a function of position and velocity but not 
of time) we start from the collisionless Boltzmann equation,

$$
\vec{v} \cdot \nabla f=\nabla V \cdot \frac{\partial f}{\partial \vec{v}},
$$

where $V=V(r)$ is a spherically symmetric gravitational potential. The density is defined by

$$
\rho=\int f d^{3} \vec{v}
$$

and it determines the gravitational potential through the Poisson equation

$$
\triangle V=4 \pi C \rho,
$$

where $C$ is a constant with dimensions of mass $x$ length ${ }^{3} /$ time $^{2}$ which for the gravitational force is the gravitational constant. A simple solution to this set of equations [Eqs. (A1) and (A3)] is given by the Maxwell-Boltzmann distribution with a singular power-law behavior in the spatial part,

$$
f(\vec{r}, \vec{v}) \sim \frac{e^{-\frac{\beta \vec{v}^{2}}{2}}}{r^{2}},
$$

where $\beta$ is a positive constant which is identified with the inverse temperature.

This solution is singular at the origin, unbounded, and has infinite mass since

$$
M(r) \propto r .
$$

The singularity at the origin can be fixed by introducing regular boundary conditions:

$$
V(0)=\text { const }, \quad V^{\prime}(0)=0,
$$

but this does not fix the asymptotic behavior. Numerical solutions of equations [Eqs. (A1) and (A3)] with boundary conditions Eqs. (A6) are solutions of isothermal spheres (since $\beta$ is constant). Far from the center at $r \gg(C \rho(0) \beta)^{-\frac{1}{2}}$ the isothermal sphere solutions approach the singular solution Eq. (A4). Although the solution is not singular at the center, it is still unbounded and has infinite mass (see, for instance, Ref. [25], pp. 480-484).

The steady-state mass distribution that solves the PoissonBoltzmann equations is singular, proportional to $\rho(r) \propto 1 / r^{2}$. To find physical solutions, it is necessary to introduce a cutoff. The assumption in the King model is that a cluster cannot keep stars whose velocity exceeds a finite escape velocity. Therefore, the velocity distribution should drop to zero at a finite limiting velocity. As a result, the cluster has a finite radius. Different cutoffs in the velocity distribution give different limiting radii. Observationally, the finite boundary is set by the tidal forces of the galaxy with which the globular cluster is associated, which become dominant at a certain distance from the cluster center. The resulting velocity distributions are simply the Maxwell-Boltzmann distributions minus a constant. Let us assume the following form at the origin:

$$
f(0, \vec{v})=\left\{\begin{array}{cc}
\alpha\left(e^{-\frac{\beta v^{2}}{2}}-e^{-\frac{\beta v_{e}(0)^{2}}{2}}\right) & v \leqslant v_{e} \\
0 & v>v_{e},
\end{array}\right.
$$

where $\alpha$ is a positive constant. This way, the fastest particles $v>v_{e}(0)$ are subtracted from the distribution. The distribution of the velocities at $r=0$ is defined by two parameters: $\beta$ (which determines the width of the distribution) and $v_{e}(0)$, the escape velocity.

According to Jeans's theorem, any steady-state solution of the collisionless Boltzmann equation is a function of the integrals of motion (see, for instance, Ref. [26], p. 220). Since energy is the integral of motion in a star cluster, the distribution function can be written as a function of the energy $E$ :

$$
f(\vec{r}, \vec{v})=f(E) .
$$

We express the cutoff as an escape energy $E_{e}$ such that $f=$ 0 for $E \geqslant E_{e}$. The escape energy corresponds to an escape velocity at a point $r$ through

$$
\begin{gathered}
E_{e}=\frac{v_{e}(r)^{2}}{2}+V(r) . \\
f(E)= \begin{cases}\alpha e^{\beta\left(V(0)-E_{e}\right)}\left[e^{-\beta\left(E-E_{e}\right)}-1\right], & E<E_{e} \\
0 & E \geqslant E_{e},\end{cases}
\end{gathered}
$$

where $\alpha$ is a positive constant.

Substituting the distribution function into Eq. (A2) and changing variables gives us

$$
\begin{gathered}
w \equiv\left(\frac{\beta}{2}\right)^{\frac{1}{2}} v, \\
\chi(r) \equiv \beta\left(E_{e}-V(r)\right) .
\end{gathered}
$$

Thus, we get

$$
\rho= \begin{cases}4 \pi \alpha e^{-\chi(0)}\left(\frac{2}{\beta}\right)^{\frac{3}{2}} I(\chi), & r<R_{e} \\ 0 & r \geqslant R_{e},\end{cases}
$$

where

$$
I(x) \equiv \int_{0}^{\sqrt{x}}\left(e^{x-w^{2}}-1\right) w^{2} d w,
$$

and $R_{e}$ is the radius of the cluster such that $v_{e}\left(R_{e}\right)=0$, and $\chi\left(R_{e}\right)=0$. In other words, any star that succeeds in reaching $R_{e}$ escapes the cluster and is no longer a part of the cluster.

When we substitute the density Eq. (A13) in the Poisson Eq. (A3), we get the fundamental equation of the King model,

$$
\frac{1}{\zeta^{2}} \frac{d}{d \zeta}\left(\zeta^{2} \frac{d \chi}{d \zeta}\right)=-\frac{I(\chi)}{I(k)}
$$

with the boundary conditions

$$
\chi(0)=k, \quad \chi^{\prime}(0)=0,
$$

where the rescaled dimensionless distance is

$$
\zeta \equiv r(4 \pi C \beta \rho(0))^{\frac{1}{2}} .
$$

The set of King solutions for the density is a one parameter family of solutions which are conveniently parameterized by $k$, the rescaled potential at the center.

Using the expression for the density Eq. (A13) and the fundamental Eq. (A15), we can write the mass in the following way:

$$
\begin{aligned}
M(r) & =4 \pi \int_{0}^{r} \rho\left(r^{\prime}\right) r^{\prime 2} d r^{\prime} \\
& =-(4 \pi \rho(0))^{-\frac{1}{2}}(\beta C)^{-\frac{3}{2}} \zeta^{2} \chi^{\prime}(\zeta)
\end{aligned}
$$




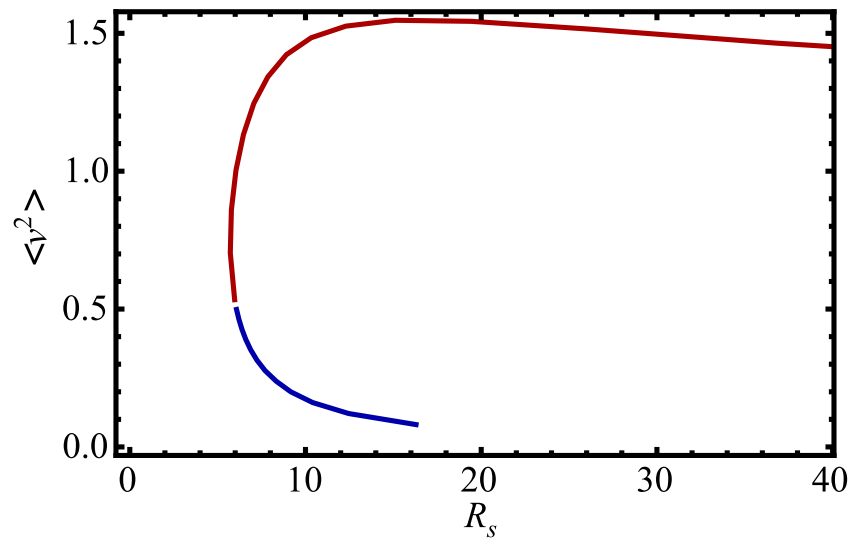

FIG. 4. The average kinetic energy (per particle) as a function of $R_{s}$ for a series of King solutions. The blue and red lines stand for the stable and unstable branches in the canonical ensemble, respectively. Note that in the limit of large clusters, the average kinetic energy is approximately independent of the cluster size.

Note that here too, in the limit $k \rightarrow \infty$, the singular solution Eq. (A4) is recovered (see Ref. [15]).

In Fig. 4, we give the average kinetic energy (per unit mass) as a function of $R_{s}$ for various King solutions. The average kinetic energy (per unit mass) $\left\langle E_{k}\right\rangle$ is given by

$$
\left\langle E_{k}\right\rangle=\frac{1}{2}\left\langle v^{2}\right\rangle=\frac{1}{2} \iint v^{2} f(\vec{r}, \vec{v}) d^{3} \vec{r} d^{3} \vec{v}
$$

when $f(\vec{r}, \vec{v})$ is normalized, so

$$
\iint v^{2} f(\vec{r}, \vec{v}) d^{3} \vec{r} d^{3} \vec{v}=1 .
$$

In the case of an isotropic distribution of velocities and spherical symmetry, we get

$$
v^{2}(r)=\int_{0}^{v_{e}(r)} v^{4} f(r, v) d v / \int_{0}^{v_{e}(r)} v^{2} f(r, v) d v .
$$

Substituting Eq. (A10) and changing variables according to Eqs. (A11) and (A12), we obtain

$$
v^{2}(r)=\frac{2}{\beta} \int_{0}^{\sqrt{\chi}}\left(e^{\chi-w^{2}}-1\right) w^{4} d w / I(\chi) .
$$

for $r<R_{e}$. Integration by parts gives us

$$
v^{2}(r)=\frac{6}{5 \beta} \frac{J_{6}(\chi(r))}{J_{4}(\chi(r))},
$$

where

$$
J_{n}(x) \equiv \int_{0}^{\sqrt{x}} w^{n} e^{-w^{2}} d w .
$$

Repeating the steps in Eqs. (A21)-(A23) with additional integration over the spatial directions gives the average kinetic energy:

$$
\left\langle E_{k}\right\rangle=\frac{1}{2}\left\langle v^{2}\right\rangle=\frac{3}{5 \beta} \frac{\int_{0}^{R_{e}} r^{2} d r e^{\chi(r)} J_{6}(\chi(r))}{\int_{0}^{R_{e}} r^{2} d r e^{\chi(r)} J_{4}(\chi(r))} .
$$

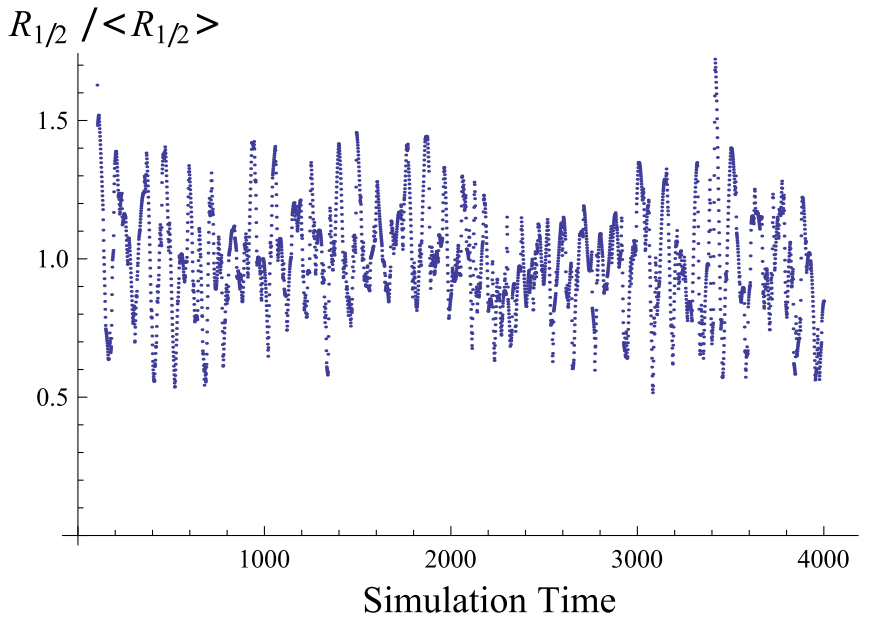

FIG. 5. The half-mass radius (normalized by its mean value) as a function of simulation time for a swarm with adaptivity $\left(R_{\mathrm{ad}}=5\right)$ and 50 individuals. The half-mass radius is defined as the minimal radius that bounds half of the individuals in the swarm. Over the sampled time period, the swarm seems to be stable for the randomly chosen initial conditions, which means that the evaporation takes place on a longer timescale.

In Fig. $4,\left\langle v^{2}\right\rangle$ is given for a series of solutions of Eq. (A15) with different values of $k$. For simplicity, we take $\beta=3 / 5$ in this figure.

The local velocity dispersion of a spherically symmetric distribution function is defined by [15]

$$
\sigma^{2}(r)=\frac{v^{2}(r)}{3}
$$

The average velocity is given by

$$
\langle v\rangle=\frac{\int_{0}^{R_{e}} r^{2} d r \int_{0}^{v_{e}(r)} v^{3} f(r, v) d v}{\int_{0}^{R_{e}} r^{2} d r \int_{0}^{v_{e}(r)} v^{2} f(r, v) d v},
$$

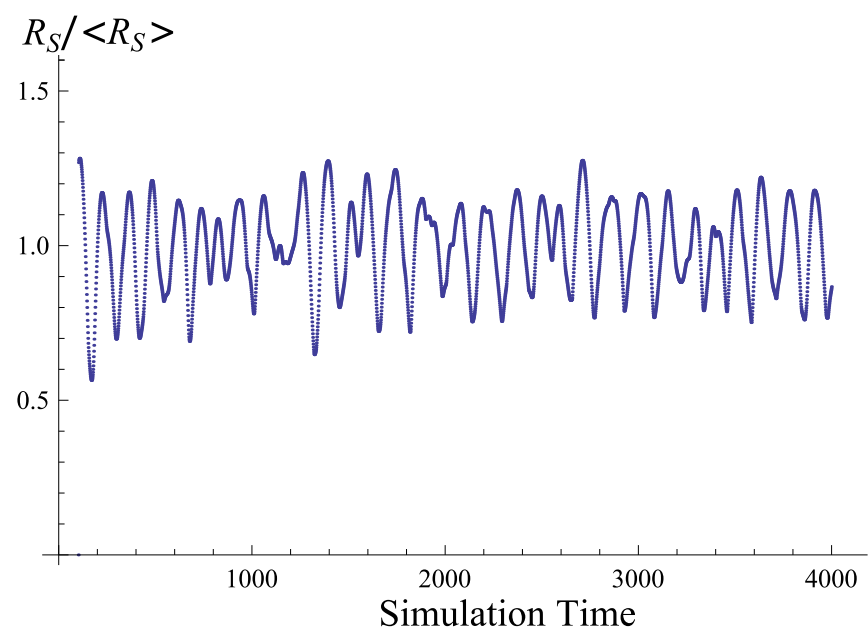

FIG. 6. The size of the swarm (normalized by its mean value) as a function of simulation time for the same swarm as in Fig. 5, where the size is defined as the mean distance from the center of the mass of the swarm. 


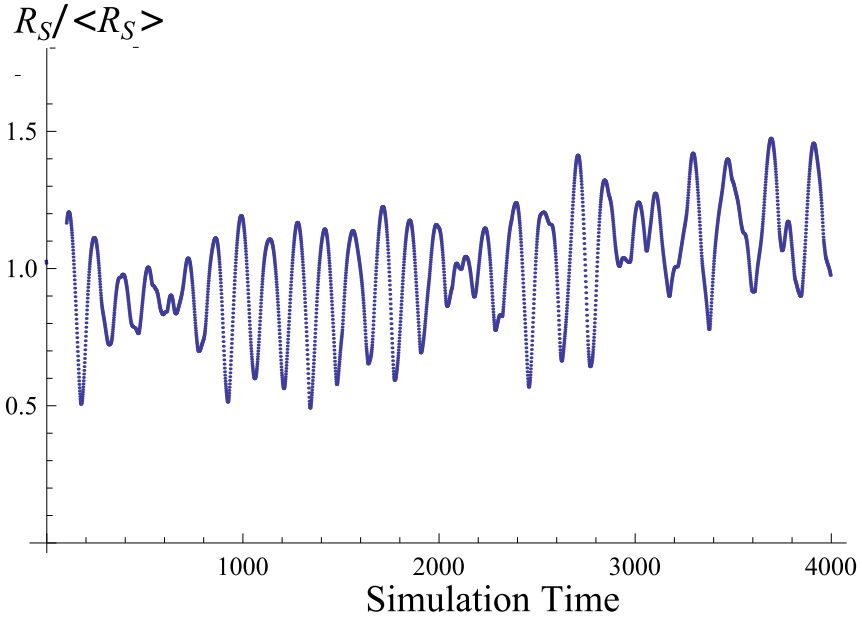

FIG. 7. The size of the swarm (normalized by its mean value) as a function of simulation time for a swarm with adaptivity $\left(R_{\mathrm{ad}}=5\right)$ and 50 individuals. Here, in this example, different initial conditions lead to evaporation at earlier times than the previous example in Fig. 6. In the examples that we considered in this paper, we did not include time segments similar to this one that showed significant evaporation.

and then we can write the dispersion normalized by the average velocity:

$$
\frac{\sigma(r)}{\langle v\rangle}=\frac{4}{3 \sqrt{5}} \sqrt{\frac{J_{6}(\chi(r))}{J_{4}(\chi(r))}} / \frac{\int_{0}^{R_{e}} r^{2} d r e^{\chi(r)} J_{5}(\chi(r))}{\int_{0}^{R_{e}} r^{2} d r e^{\chi(r)} J_{4}(\chi(r))} .
$$

\section{APPENDIX B: THE TIME EVOLUTION OF THE SWARM IN THE SIMULATION}

Here we show a typical oscillatory behavior as a function of time of the half-mass radius (Fig. 5) and the size of the swarm (defined as the mean distance from the center of the swarm) in Fig. 6 for initial conditions that do not cause evaporation (over the time segment that is presented here). In Fig. 7, we show that the same swarm with different initial conditions evaporates over the same time segment (Fig. 7). Such cases were not considered as stable in this paper and were ignored.

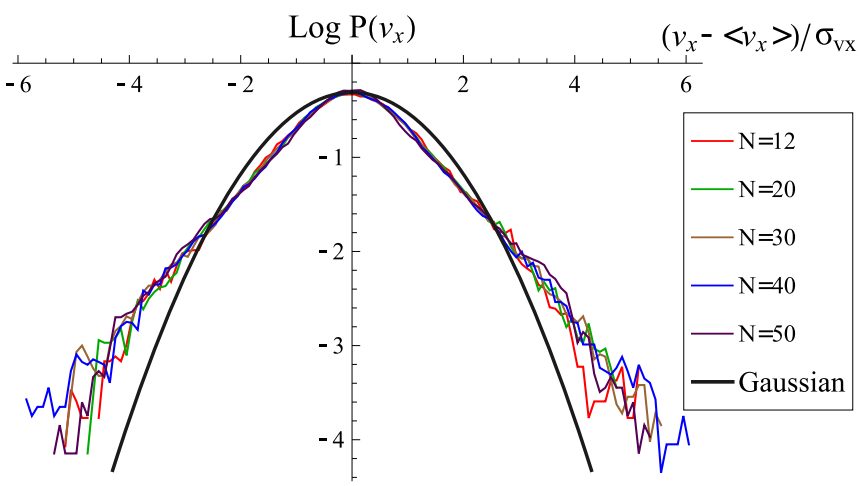

FIG. 8. The distribution of the $x$ component of the velocity normalized by its standard deviation for different simulated swarms with adaptivity $\left(R_{\mathrm{ad}}=5\right)$. A reference Gaussian curve is shown in black.

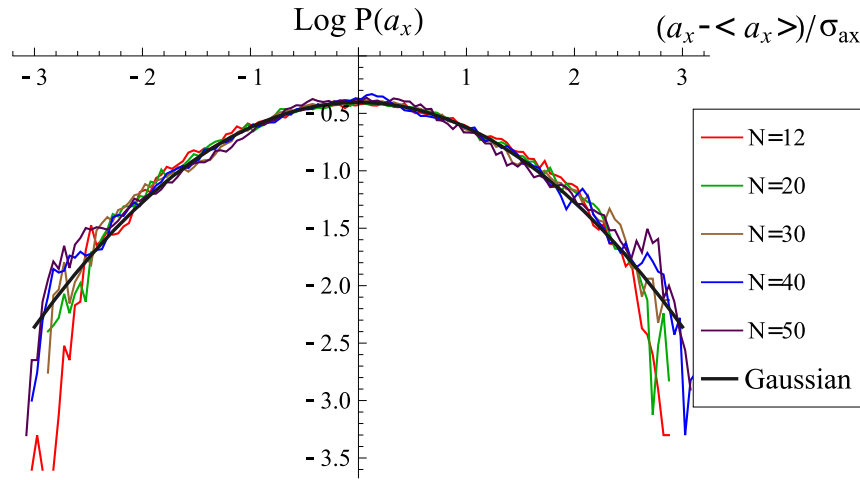

FIG. 9. The distribution of the $x$ component of the acceleration normalized by its standard deviation for different simulated swarms with adaptivity $\left(R_{\mathrm{ad}}=5\right)$. The acceleration distribution is bounded and cannot reach values that are too far from the average value. This is a result of the addition of the adaptivity and the softening parameter $\epsilon$ to the simulation, which are needed to slow down the evaporation of the swarm. A reference Gaussian curve is shown in black.

\section{APPENDIX C: THE VELOCITY AND ACCELERATION DISTRIBUTIONS}

The distribution of a single component of the velocity in the simulation is given in Fig. 8 for various swarm sizes. In Fig. 9, we give the similar distribution of a single component of the acceleration. The structure of the tails is similar to the distributions that were obtained from the laboratory data [4]. (a)

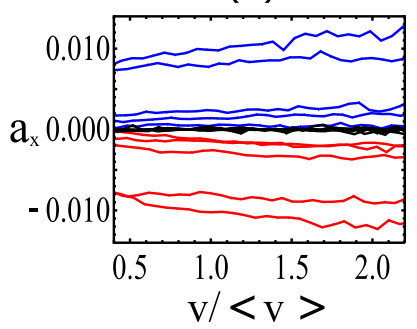

(c)

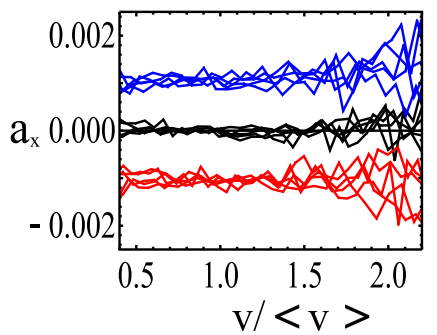

(b)

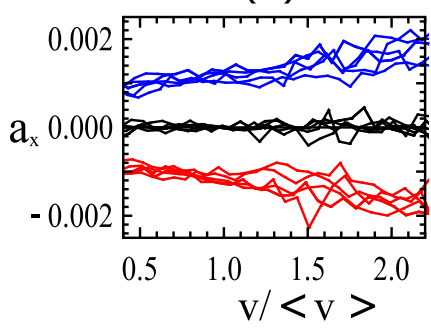

(d)

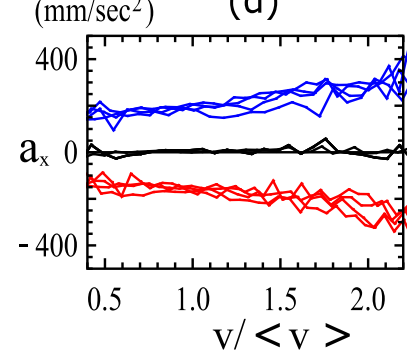

FIG. 10. The mean value of a single component of the acceleration versus the speed (normalized by the average speed) of the left hemisphere (blue), right hemisphere (red), and both hemispheres (black). The overall acceleration (black lines) is close to zero, as required by symmetry. Data are shown for (a) $\epsilon$ gravity for different values of $R_{s}$ (with $R_{s}=16.23,3.16,19.53,18.37,30.10$ from top to bottom), (b) adaptive gravity, (c) adaptive gravity with repulsion, and (d) observational data. 
(a)

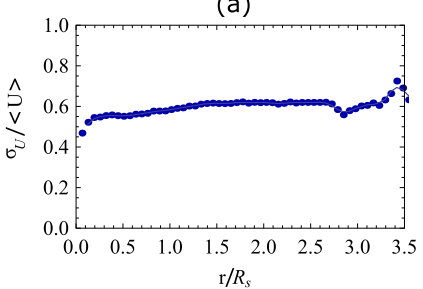

(c)

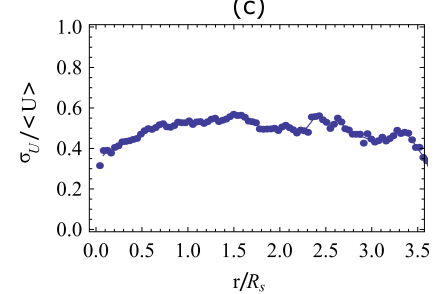

(b)

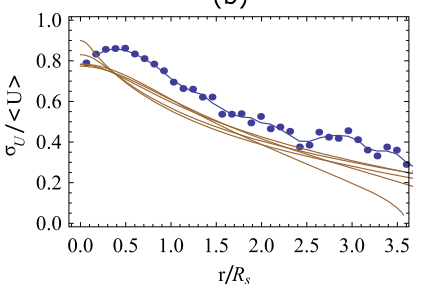

(d)

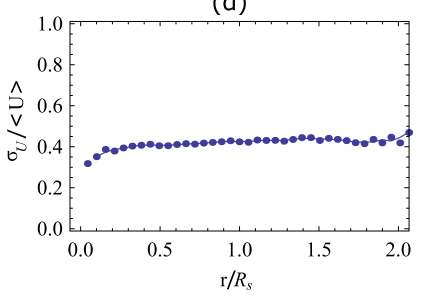

FIG. 11. The standard deviation of the speed $\left(\sigma_{U}\right)$ normalized by the average speed in the swarm as a function of the distance from the center of mass normalized by the swarm size $R_{s}$. Data are shown for (a) laboratory observations, (b) simulation of $\epsilon$-gravity [note that the brown curves correspond to a family of King solutions computed numerically according to Eq. (A28)], (c) adaptive gravity, and (d) adaptive gravity with repulsion.

\section{APPENDIX D: THE RELATION BETWEEN MEAN ACCELERATION AND SPEED}

It was observed in Ref. [17] that if we take the mean acceleration of the right or left hemisphere of the swarm separately, its absolute value is a monotonically increasing function of the speed. The observational data from Ref. [17] for various sizes of swarms is reproduced here in Fig. 10(d), where the speed is normalized with respect to its mean value. Comparing with the simulation results [for $\epsilon$ gravity in Fig. 10(a), adaptivity in Fig. 10(b), and adaptivity with short-range repulsion in Fig. 10(c)] for swarms of different sizes, we see that in the cases with adaptivity (with and without repulsion), all the graphs collapse at low velocities to have approximately the same acceleration, whereas in the $\epsilon$-gravity case there are various values of acceleration at low velocities that do not agree with the observational data in Fig. 10(d). One can also see that with repulsion there is more noise at high velocities.
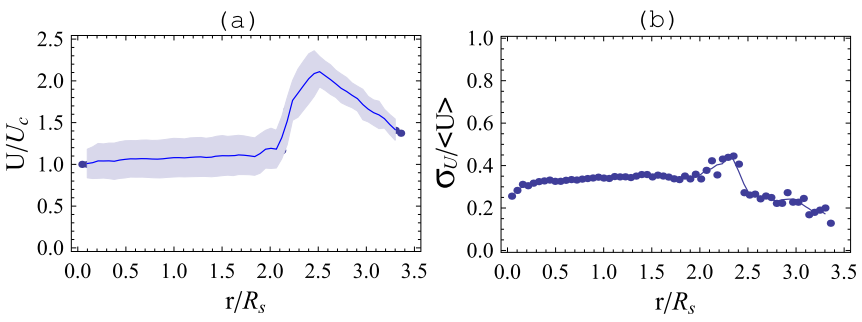

FIG. 12. (a) Mean speed (normalized by the speed at the swarm center) and (b) its standard deviation (normalized by the average speed) as functions of the distance from the swarm center for simulations with adaptvity and repulsion. For $r>2 R_{s}$, the velocities can become up to two times larger and the standard deviation can become two times lower. At the same time, the density is less than $10^{-5}$ of the density at the center (Fig. 1). Therefore, this regime likely describes particles that were repelled out of the swarm at high velocities (higher than the escape velocity).

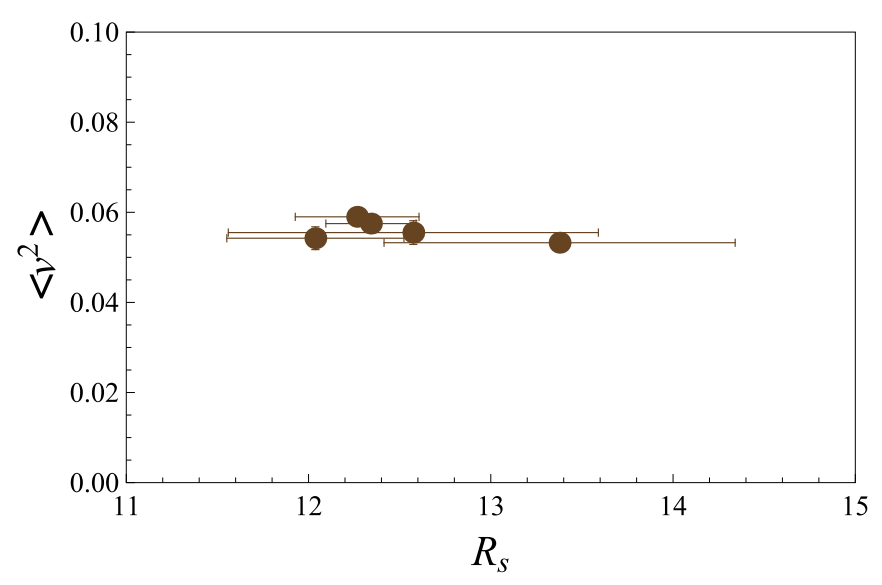

FIG. 13. The kinetic energy as a function of the size of the swarm $R_{s}$ for cases with adaptivity (Fig. 2). Here the size of the swarm does not change much but the number of individuals changes from 12 to 48 (Fig. 2).

The agreement that we see here between adaptivity and the observational data suggests that adaptive gravity naturally explains the dependence of the acceleration on the mean velocity, without the need to invoke any additional explicit velocity-dependent forces [17].

\section{APPENDIX E: THE STANDARD DEVIATION OF THE SPEED IN THE SIMULATIONS AND THE LABORATORY MEASUREMENTS}

In addition to the uniformity of the average velocity across the midge swarm in the observational data (Fig. 3), we observe the same uniformity for the standard deviation of the speed (Fig. 11). The addition of adaptivity to the model recovers this behavior, as seen in Fig. 11.

In Fig. 12, we see the full range of velocity profiles and standard deviations of the speed for the simulations that include adaptivity and repulsion. For $r>2 R_{s}$, the velocities are found to increase to very high values and the density is very low. This region corresponds to particles that were expelled out of the swarm at high velocities due to the short-range

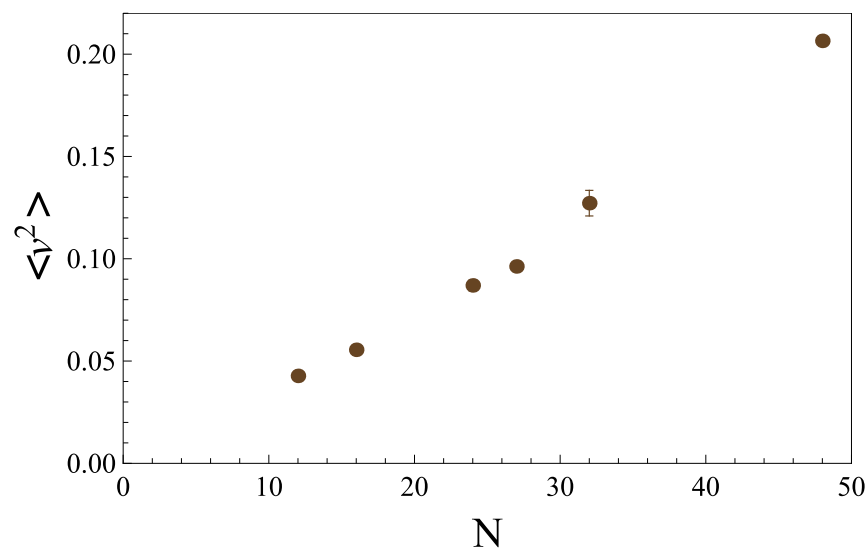

FIG. 14. The kinetic energy as a function of the number of individuals $N$ in the simulation for the cases without adaptivity (Fig. 15). 

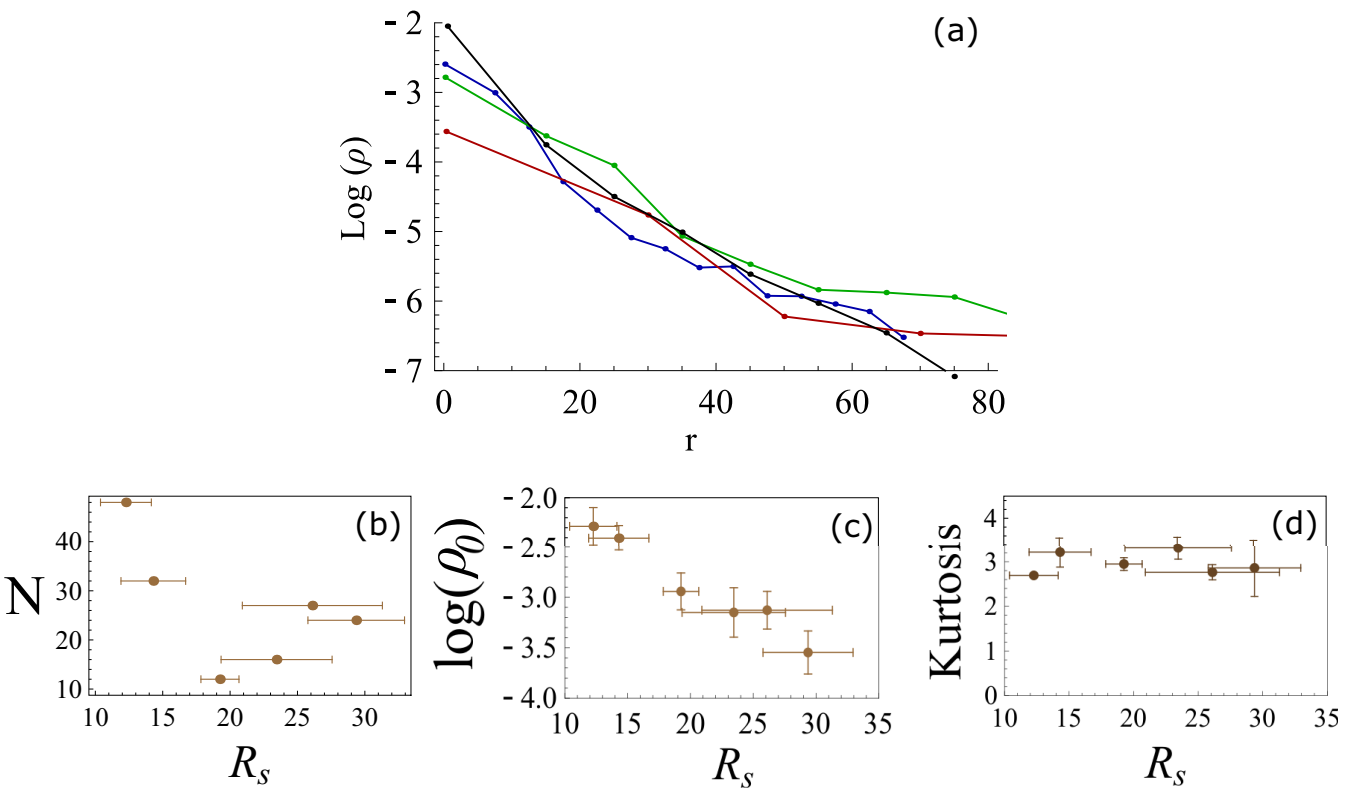

FIG. 15. (a) Density profiles of simulated clusters with the softened-gravity ("epsilon gravity") model. Data are shown for cases with $N=48, R_{s}=9.29$ (black), $N=27, R_{s}=26.08$ (green), $N=16, R_{s}=30$ (red), and $N=12, R_{s}=16.4$ (blue). (b)-(d) The total number of midges, the density at the center, and the kurtosis as functions of the size of the swarm for the "epsilon gravity" model (using $\epsilon^{2}=15$ ).

repulsive interactions. We therefore do not consider this regime to be meaningful with respect to the properties of swarms.

\section{APPENDIX F: KINETIC ENERGY IN THE SIMULATION}

The kinetic energy of each midge in the laboratory measurements is approximately constant and does not depend on the density or the size of the swarm. In the simulations, since we are interested only in the mutual forces, we do not require constant speed for the particles, and therefore the kinetic energy per individual is different for various numbers of particles and initial conditions. We chose initial conditions that give us approximately the same kinetic energy per individual in the case of simulations with adaptivity and adaptivity with repulsion (within about 10\%, see Fig. 13). Without adaptivity, we could not significantly change the kinetic energy by varying the initial conditions, and so it was determined mainly by the number of particles in the simulation (Fig. 14).

\section{APPENDIX G: THE SIMULATION WITH SOFTENED GRAVITY ("EPSILON GRAVITY")}

To avoid high accelerations due to close encounters ("slingshots") and thus evaporation of the cluster, we included a softening parameter $\epsilon$ to the gravitational force [Eq. (1)]. The particles in the simulation still developed high accelerations in close encounters relative to simulations with adaptivity since adaptivity itself contributed to the decrease of accelerations by $\epsilon^{2} /\left(R_{\mathrm{ad}}^{2}+\epsilon^{2}\right)$, and to compare the simulations in the two cases (with and without adaptivity) we have to reduce the accelerations in the " $\epsilon$-gravity" case by this constant factor. The results in Fig. 15 were obtained in this way. In Fig. 15(a), density profiles for various sizes of clusters are given (where $\epsilon^{2}=15$ ) and in Figs. 15(b)-15(d) we show three characteristic quantities of the clusters: the number of particles, the density at the center, and the kurtosis. Here and throughout the paper we truncated the tails at $r \sim 3 R_{s}$ in the calculation of the kurtosis.
[1] J. Krause and G. D. Ruxton, Living in Groups (Oxford University Press, Oxford, 2002).

[2] D. Sumpter, Collective Animal Behavior (Princeton University Press, Princeton, 2010).

[3] A. Okubo, Adv. Biophys. 22, 1 (1986).

[4] D. H. Kelley and N. T. Ouellette, Sci. Rep. 3, 1073 (2013).

[5] A. Attanasi, A. Cavagna, L. Del Castello, I. Giardina, S. Melillo, L. Parisi, O. Pohl, B. Rossaro, E. Shen, E. Silvestri, and M. Viale, Phys. Rev. Lett. 113, 238102 (2014).

[6] R. Ni, J. G. Puckett, E. R. Dufresne, and N. T. Ouellette, Phys. Rev. Lett. 115, 118104 (2015).
[7] M. Sinhuber, K. van der Vaart, and N. T. Ouellette, J. R. Soc. Interface 16, 20180739 (2019).

[8] K. van der Vaart, M. Sinhuber, A. M. Reynolds, and N. T. Ouellette, Sci. Adv. 5, eaaw9305 (2019).

[9] A. Aldersley, A. Champneys, M. Homer, N. W. Bode, and D. Robert, Curr. Biol. 27, R1208 (2017).

[10] D. Gorbonos, R. Ianconescu, J. G. Puckett, R. Ni, N. T. Ouellette, and N. S. Gov, New J. Phys. 18, 073042 (2016).

[11] D. Gorbonos and N. S. Gov, Phys. Rev. E 95, 042405 (2017).

[12] J. Seuer, E. J. Tuck, and D. Robert, J. Acoust. Soc. Am. 118, 530 (2005). 
[13] T. Vicsek, A. Czirók, E. Ben-Jacob, I. Cohen, and O. Shochet, Phys. Rev. Lett. 75, 1226 (1995).

[14] I. King, Astron. J. 71, 64 (1966).

[15] P.-H. Chavanis, M. Lemou, and F. Méhats, Phys. Rev. D 91, 063531 (2015).

[16] J. G. Puckett and N. T. Ouellette, J. R. Soc. Interface 11, 20140710 (2014).

[17] A. M. Reynolds, M. Sinhuber, and N. T. Ouellette, Eur. Phys. J. E 40, 46 (2017).

[18] M. Sinhuber, K. van der Vaart, R. Ni, J. G. Puckett, D. H. Kelley, and N. T. Ouellette, Sci. Data 6, 190036 (2019).

[19] S. J. Aarseth, in Multiple Time Scales, edited by J. U. Brackbill and B. I. Cohen (Academic Press, Orlando, 1985).
[20] O. Shoval, L. Goentoro, Y. Hart, A. Mayo, E. Sontag, and U. Alon, Proc. Natl. Acad. Sci. USA 107, 15995 (2010).

[21] J. G. Puckett, D. H. Kelley, and N. T. Ouellette, Sci. Rep. 4, 4766 (2014).

[22] J. Binney and S. Tremaine, Galactic Dynamics (Princeton University Press, Princeton, 2011).

[23] A. M. Reynolds and N. T. Ouellette, Sci. Rep. 6, 30515 (2016).

[24] A. M. Reynolds, Phys. Biol. 16, 046002 (2019).

[25] T. Padmanabhan, Theoretical Astrophysics: Volume 1, Astrophysical Processes (Cambridge University Press, Cambridge, UK, 2000).

[26] J. Binney and S. Tremaine, Galactic Dynamics (Princeton University Press, New Jersey, USA, 2011). 